MATEMATIKA, 2017, Volume 33, Number 2, 177-189

(C) Penerbit UTM Press. All rights reserved

\title{
Direct and inverse theorems of approximation theory in $L^{2}\left(\mathbb{R}^{d}, w_{l}(x) d x\right)$
}

\author{
${ }^{1}$ Radouan Daher, ${ }^{2}$ Salah El Ouadih and ${ }^{3}$ Mohamed El Hamma \\ $1,2,3$ Department of Mathematics, Faculty of Sciences Aïn Chock, \\ University Hassan II, Casablanca, Morocco \\ e-mail: ${ }^{1}$ rjdaher024@gmail.com, ${ }^{2}$ salahwadih@gmail.com, ${ }^{3}$ m-elhamma@yahoo.fr
}

\begin{abstract}
In this paper, we prove analogues of direct and some inverse theorems for the Dunkl harmonic analysis, using the function with bounded spectrum and generalized spherical mean operator.
\end{abstract}

Keywords Generalized continuity modulus; Bernstein theorem; Jackson theorem; best approximation.

AMS Mathematics Subject Classification 42B37, 42B10

\section{Introduction}

Yet by the year 1912, S. Bernstein obtained the estimate inverse to Jakson's inequality in the space of continuous functions for some special cases [1], later Stechkin [2], Timan [3], proved such inverse estimates, including the case of the space $L^{p}, 1<p<\infty$.

Approximation problems for functions in the space $L^{2}\left(\mathbb{R}^{d}, w_{l}(x) d x\right)$, where $w_{l}$ is a weight function invariant under the action of an associated reflection groups, using the function with bounded spectrum, are studied in this paper. Applying the Dunkl transform, Dunkl Laplacian operator and generalized spherical mean operator, we obtain analogs of the Bernstein inequality for function with bounded spectrum, direct and inverse theorem of Jackson type [4],[2],[5], where the modulus of smoothness is constructed on the basis of generalized spherical mean operator.

\section{The Dunkl transform and its basic properties}

Dunkl [3] defined a family of first-order differential-difference operators related to some reflection groups. These operators generalize in a certain manner the usual differentiation and have gained considerable interest in various fields of mathematics and also in physical applications. The theory of Dunkl operators provides generalizations of various multivariable analytic structures. Among others, we cite the exponential function, the Fourier transform and the translation operator. For more details about these operators see [6], [7], [1] and [8].

Let $R$ be a root system in $\mathbb{R}^{d}, \mathrm{~W}$ the corresponding reflection group, $R_{+}$a positive subsystem of $R$ and $l$ a non-negative and $\mathrm{W}$-invariant function defined on $R$. The Dunkl operator is defined for $f \in C^{1}\left(\mathbb{R}^{d}\right)$ by

$$
D_{j} f(x)=\frac{\partial f}{\partial x_{j}}(x)+\sum_{\alpha \in R_{+}} l(\alpha) \alpha_{j} \frac{f(x)-f\left(\sigma_{\alpha}(x)\right)}{\langle\alpha, x\rangle}, x \in \mathbb{R}^{d} .
$$

Here $\langle$,$\rangle is the usual Euclidean scalar product on \mathbb{R}^{d}$ with the associated norm $|$.$| and \sigma_{\alpha}$ the reflection with respect to the hyperplane $H_{\alpha}$ orthogonal to $\alpha$. We consider the weight 
function

$$
w_{l}(x)=\prod_{\alpha \in R_{+}}|\langle\alpha, x\rangle|^{2 l(\alpha)},
$$

where $w_{l}$ is $\mathrm{W}$-invariant and homogeneous of degree $2 \gamma$ where

$$
\gamma=\sum_{\alpha \in R_{+}} l(\alpha)
$$

We let $\eta$ be the normalized surface measure on the unit sphere $\mathbb{S}^{d-1}$ in $\mathbb{R}^{d}$ and set

$$
d \eta_{l}(y)=w_{l}(y) d \eta(y)
$$

Then $\eta_{l}$ is a W-invariant measure on $\mathbb{S}^{d-1}$, and we let $d_{l}=\eta_{l}\left(\mathbb{S}^{d-1}\right)$.

The Dunkl kernel $E_{l}$ on $\mathbb{R}^{d} \times \mathbb{R}^{d}$ has been introduced by Dunkl in [9]. For $y \in \mathbb{R}^{d}$ the function $x \mapsto E(x, y)$ can be viewed as the solution on $\mathbb{R}^{d}$ of the following initial problem:

$$
\left\{\begin{aligned}
D_{j} u(x, y)=y_{j} u(x, y), & \text { if } 1 \leq j \leq d \\
u(0, y)=0, & \text { for all } y \in \mathbb{R}^{d}
\end{aligned}\right.
$$

This kernel has a unique holomorphic extension to $\mathbb{C}^{d} \times \mathbb{C}^{d}$.

Rösler has proved in [8] the following integral representation for the Dunkl kernel,

$$
E_{l}(x, z)=\int_{\mathbb{R}^{d}} e^{\langle y, z\rangle} d \mu_{x}(y), x \in \mathbb{R}^{d}, z \in \mathbb{C}^{d},
$$

where $\mu_{x}$ is a probability measure on $\mathbb{R}^{d}$ with support in the closed ball $B(0,|x|)$ of center 0 and radius $|x|$.

Proposition $1[6]$ Let $z, w \in \mathbb{C}^{d}$ and $\lambda \in \mathbb{C}$

(i) $E_{l}(z, 0)=1, E_{l}(z, w)=E_{l}(w, z), E_{l}(\lambda z, w)=E_{l}(z, \lambda w)$.

(ii) For all $\nu=\left(\nu_{1}, \ldots, \nu_{d}\right) \in \mathbb{N}, x \in \mathbb{R}^{d}, z \in \mathbb{C}^{d}$, we have

$$
\left|D_{z}^{\nu} E_{k}(x ; z)\right| \leq|x|^{|\nu|} \exp (|x||\operatorname{Re} z|,
$$

where

$$
D_{z}^{\nu}=\frac{\partial^{|\nu|}}{\partial_{z_{1}}^{|\nu|} \ldots \partial_{z_{d}}^{|\nu|}},|\nu|=\nu_{1}+\ldots+\nu_{d}
$$

In particular $\left|D_{z}^{\nu} E_{l}(i x ; z)\right| \leq|x|^{|\nu|}$ for all $x, z \in \mathbb{R}^{d}$.

We denote by $L_{l}^{2}\left(\mathbb{R}^{d}\right)=L^{2}\left(\mathbb{R}^{d}, w_{l}(x) d x\right)$ the space of measurable functions on $\mathbb{R}^{d}$ such that

$$
\|f\|_{2, l}=\left(\int_{\mathbb{R}^{d}}|f(x)|^{2} w_{l}(x) d x\right)^{\frac{1}{2}},
$$

and $D_{l}$ the Dunkl Laplacian defined by

$$
D_{l}=\sum_{i=1}^{d} D_{j}^{2} .
$$


The scalar product in the Hilbert space $L_{l}^{2}\left(\mathbb{R}^{d}\right)$ obeys the formula

$$
(f, g):=\int_{\mathbb{R}^{d}} \overline{f(x)} g(x) w_{l}(x) d x, \quad f, g \in L_{l}^{2}\left(\mathbb{R}^{d}\right) .
$$

By the partial integration one can verify the correlation

$$
\left(D_{l} f, g\right)=\left(g, D_{l} f\right)
$$

for any functions $f, g \in \mathcal{D}$ ( $\mathcal{D}$ denotes the set of infinitely differentiable functions with a compact support).

As usual, we endow the space $\mathcal{D}$ with a topology; this turns it into a topological vector space [10]. Let $\mathcal{D}^{\prime}$ stand for the set of generalized functions, i.e., linear continuous functionals on the space $\mathcal{D}$. We denote the value of a functional $f \in \mathcal{D}^{\prime}$ on a function $\varphi \in \mathcal{D}$ by $\langle f, \varphi\rangle$. The space $L_{l}^{2}\left(\mathbb{R}^{d}\right)$ is embedded into $\mathcal{D}^{\prime}$, provided that for $f \in L_{l}^{2}\left(\mathbb{R}^{d}\right)$ and $\varphi \in \mathcal{D}$ we put

$$
\langle f, \varphi\rangle:=\int_{\mathbb{R}^{d}} f(x) \varphi(x) w_{l}(x) d x .
$$

One can extend (in a natural way) the action of the Dunkl Laplacian operator $D_{l}$ onto the space of generalized functions $\mathcal{D}^{\prime}$, putting

$$
\left\langle D_{l} f, \varphi\right\rangle:=\left\langle f, D_{l} \varphi\right\rangle, \quad f \in \mathcal{D}^{\prime}, \varphi \in \mathcal{D} .
$$

In particular, the action of the operator $D_{l} f$ is defined for any function $f \in L_{l}^{2}\left(\mathbb{R}^{d}\right)$ but, generally speaking, $D_{l} f$ is a generalized function.

The Dunkl transform is defined for $f \in L_{l}^{1}\left(\mathbb{R}^{d}\right)=L^{1}\left(\mathbb{R}^{d}, w_{l}(x) d x\right)$

$$
\mathcal{F}(f)(\xi)=\widehat{f}(\xi)=c_{l}^{-1} \int_{\mathbb{R}^{d}} f(x) E_{l}(-i \xi, x) w_{l}(x) d x,
$$

where the constant $c_{l}$ is given by

$$
c_{l}=\int_{\mathbb{R}^{d}} e^{-\frac{|x|^{2}}{2}} w_{l}(z) d z .
$$

The inverse Dunkl transform is defined by the formula

$$
f(x)=\int_{\mathbb{R}^{d}} \widehat{f}(\xi) E_{l}(i x, \xi) w_{l}(\xi) d \xi, x \in \mathbb{R}^{d} .
$$

From [11], we have that if $f \in L_{l}^{2}\left(\mathbb{R}^{d}\right)$

$$
\widehat{D_{l} f}(\xi)=-|\xi|^{2} \widehat{f}(\xi) .
$$

The Dunkl transform shares several properties with its counterpart in the classical case. We mention here, in particular that Parseval theorem holds in $L_{l}^{2}\left(\mathbb{R}^{d}\right)$. As in the classical case, a generalized translation operator is defined in the Dunkl $[5,12]$. Namely, for $f \in L_{l}^{2}\left(\mathbb{R}^{d}\right)$ and $x \in \mathbb{R}^{d}$ we define $\tau_{x}(f)$ to be the unique function in $L_{l}^{2}\left(\mathbb{R}^{d}\right)$ satisfying

$$
\widehat{\tau_{x} f}(y)=E_{l}(i x, y) \widehat{f}(y) \quad \text { a.e } \quad y \in \mathbb{R}^{d} .
$$


Form to Parseval theorem and proposition 1.1, we see that

$$
\left\|\tau_{x} f\right\|_{2, l} \leq\|f\|_{2, l} \text { for all } \quad x \in \mathbb{R}^{d} .
$$

For $\alpha>\frac{-1}{2}$, let $j_{\alpha}(x)$ be a normalized Bessel function of the first kind, i.e.,

$$
j_{\alpha}(x)=\frac{2^{\alpha} \Gamma(\alpha+1) J_{\alpha}(x)}{x^{\alpha}},
$$

where $J_{\alpha}(x)$ is a Bessel function of the first kind [13].

The function $J_{\alpha}(x)$ is infinitely differentiable, $J_{\alpha}(0)=1$.

Proposition 2 ([14] or [15]) For $x \in \mathbb{R}$ the following inequalities are fulfilled

(i) $\left|j_{\alpha}(x)\right| \leq 1$.

(ii) $\left|1-j_{\alpha}(x)\right| \geq c_{0}$ with $|x| \geq 1$, where $c_{0}>0$ is a certain constant which depend only on $\alpha$.

(iii) $\left|1-j_{\alpha}(x)\right| \leq c_{1} x^{2}$, where $c_{1}$ is a constant.

The generalized spherical mean value of $f \in L_{l}^{2}\left(\mathbb{R}^{d}\right)$ is defined by

$$
M_{h} f(x)=\frac{1}{d_{l}} \int_{\mathbb{S}^{d-1}} \tau_{x} f(h y) d \eta_{l}(y), x \in \mathbb{R}^{d}, h>0 .
$$

We have

$$
\left\|M_{h} f\right\|_{2, l} \leq\|f\|_{2, l} .
$$

Proposition $3([16])$ Let $f \in L_{l}^{2}\left(\mathbb{R}^{d}\right)$ and $f i x h>0$. Then $M_{h} f \in L_{l}^{2}\left(\mathbb{R}^{d}\right)$ and

$$
\widehat{M_{h} f}(\xi)=j_{\gamma+\frac{d}{2}-1}(h|\xi|) \widehat{f}(\xi), \xi \in \mathbb{R}^{d} .
$$

Let the function $f \in L_{l}^{2}\left(\mathbb{R}^{d}\right)$. We define differences of the order $k(k=1,2, \ldots)$ with a step $h>0$.

$$
\Delta_{h}^{k} f(x)=\left(I-M_{h}\right)^{k} f(x)
$$

where I is the unit operator.

For any positive integer $k$, we define the generalized module of smoothness of the $k$ th order by the formula

$$
\omega_{k}(f, \delta)_{2, l}=\sup _{0<h \leq \delta}\left\|\Delta_{h}^{k} f\right\|_{2, l}, \delta>0
$$

Let $W_{2, l}^{k}$ be the Sobolev space constructed by the operator $D_{l}$, i.e.,

$$
W_{2, l}^{k}=\left\{f \in L_{l}^{2}\left(\mathbb{R}^{d}\right): D_{l}^{j} f \in L_{l}^{2}\left(\mathbb{R}^{d}\right) ; j=1,2, \ldots, k\right\}
$$

where $D_{l}^{0} f=f, D_{l}^{j} f=D_{l}\left(D_{l}^{j-1} f\right)$.

For any $f \in L_{l}^{2}\left(\mathbb{R}^{d}\right)$ and any number $\nu>0$, let us define the function

$$
P_{\nu}(f)(x)=\mathcal{F}^{-1}\left(\widehat{f}(\xi) \chi_{\nu}(\xi)\right)
$$


where $\chi_{\nu}(\xi)=1$ if $|\xi| \leq \nu$ and $\chi_{\nu}(\xi)=0$ if $|\xi|>\nu, \mathcal{F}^{-1}$ is the inverse Dunkl transform. One can easily prove that the function $P_{\nu}(f)(x)$ is infinitely differentiable and belongs to all classes $W_{2, l}^{k}, k=1,2, \ldots$

A function $f \in L_{l}^{2}\left(\mathbb{R}^{d}\right)$ is called a function with bounded spectrum of order $\nu>0$ if $\widehat{f}(\xi)=0$ for $|\xi|>\nu$. The set of all such functions is denoted by $\mathcal{I}_{\nu}$.

The best approximation of a function $f \in L_{l}^{2}\left(\mathbb{R}^{d}\right)$ by functions in $\mathcal{I}_{\nu}$ is the quantity

$$
E_{\nu}(f)_{2, l}:=\inf _{g \in \mathcal{I}_{\nu}}\|f-g\|_{2, l} .
$$

\section{Bernstein's inequality and Jackson's direct theorems}

\section{Bernstein's Theorem 1}

If $f \in \mathcal{I}_{\nu}$, then $D_{l} f \in \mathcal{I}_{\nu}$ and

$$
\left\|D_{l} f\right\|_{2, l} \leq \nu^{2}\|f\|_{2, l}
$$

Proof

From equality (1), we have $D_{l} f \in \mathcal{I}_{\nu}$ if $f \in \mathcal{I}_{\nu}$

Formula (1) and Parseval theorem gives

$$
\begin{aligned}
\left\|D_{l} f\right\|_{2, l}^{2} & =\int_{\mathbb{R}^{d}}\left|D_{l} f(x)\right|^{2} w_{l}(x) d x \\
& =\int_{\mathbb{R}^{d}}\left|\widehat{D_{l} f}(\xi)\right|^{2} w_{l}(\xi) d \xi \\
& =\int_{|\xi| \leq \nu}|\xi|^{4}|\widehat{f}(\xi)|^{2} w_{l}(\xi) d \xi \\
& \leq \nu^{4} \int_{\mathbb{R}^{d}}|\widehat{f}(\xi)|^{2} w_{l}(\xi) d \xi \\
& =\nu^{4} \int_{\mathbb{R}^{d}}|f(x)|^{2} w_{l}(x) d x \\
& =\nu^{4}\|f\|_{2, l}^{2} .
\end{aligned}
$$

Therefore

$$
\left\|D_{l} f\right\|_{2, l} \leq \nu^{2}\|f\|_{2, l}
$$

Jackson's Theorem 2

Suppose that $f \in W_{2, l}^{m}(m=1,2, \ldots)$, then

$$
E_{\nu}(f)_{2, l} \leq c_{2} \nu^{-2 m} \omega_{k}\left(D_{l}^{m} f, 1 / \nu\right)_{2, l},
$$

for all $\nu>0$, where $c_{2}=c_{0}^{-(k+m)} c_{1}^{m}$ is a constant. 


\section{Proof}

Using the Parseval equality, we have

$$
\begin{aligned}
\left\|f-P_{\nu}(f)\right\|_{2, l}^{2} & =\int_{\mathbb{R}^{d}}\left|f(x)-P_{\nu}(f)(x)\right|^{2} w_{l}(x) d x \\
& =\int_{\mathbb{R}^{d}}\left|\widehat{f}(\xi)-\widehat{P_{\nu}(f)}(\xi)\right|^{2} w_{l}(\xi) d \xi \\
& =\int_{\mathbb{R}^{d}}\left|\left(1-\chi_{\nu}(\xi)\right) \widehat{f}(\xi)\right|^{2} w_{l}(\xi) d \xi .
\end{aligned}
$$

Therefore

$$
\left\|f-P_{\nu}(f)\right\|_{2, l}^{2}=\int_{|\xi|>\nu}|\widehat{f}(\xi)|^{2} w_{l}(\xi) d \xi
$$

By Proposition 2, we have

$$
\left|1-j_{\gamma+\frac{d}{2}-1}(|\xi| / \nu)\right| \geq c_{0}
$$

for $|\xi| \geq \nu$.

Therefore, from (3) and Parseval equality we deduce that

$$
\begin{aligned}
\left\|f-P_{\nu}(f)\right\|_{2, l}^{2} & \leq \frac{1}{c_{0}^{2(k+m)}} \int_{\mathbb{R}^{d}}\left(1-j_{\gamma+\frac{d}{2}-1}(|\xi| / \nu)\right)^{2(k+m)}|\widehat{f}(\xi)|^{2} w_{l}(\xi) d \xi \\
& =\frac{1}{c_{0}^{2(k+m)}} \int_{\mathbb{R}^{d}}\left|\mathcal{F}\left(\left(I-M_{1 / \nu}\right)^{k+m} f\right)(\xi)\right|^{2} w_{l}(\xi) d \xi \\
& =\frac{1}{c_{0}^{2(k+m)}} \int_{\mathbb{R}^{d}}\left|\left(I-M_{1 / \nu}\right)^{k+m} f(x)\right|^{2} w_{l}(x) d x \\
& =\frac{1}{c_{0}^{2(k+m)}}\left\|\left(I-M_{1 / \nu}\right)^{k+m} f\right\|_{2, l}^{2} .
\end{aligned}
$$

Therefore

$$
\left\|f-P_{\nu}(f)\right\|_{2, l} \leq c_{0}^{-(k+m)}\left\|\left(I-M_{1 / \nu}\right)^{k+m} f\right\|_{2, l} .
$$

Proposition 2, Parseval equality and formula (1) show that

$$
\begin{aligned}
\left\|\left(I-M_{1 / \nu}\right) f\right\|_{2, l}^{2} & =\int_{\mathbb{R}^{d}}\left|\left(I-M_{1 / \nu}\right) f(x)\right|^{2} w_{l}(x) d x \\
& =\int_{\mathbb{R}^{d}}\left|\mathcal{F}\left(\left(I-M_{1 / \nu}\right) f\right)(\xi)\right|^{2} w_{l}(\xi) d \xi \\
& =\int_{\mathbb{R}^{d}}\left(1-j_{\gamma+\frac{d}{2}-1}(|\xi| / \nu)\right)^{2}|\widehat{f}(\xi)|^{2} w_{l}(\xi) d \xi \\
& \leq\left. c_{1}^{2} \nu^{-4} \int_{\mathbb{R}^{d}}|\xi|\right|^{4}|\widehat{f}(\xi)|^{2} w_{l}(\xi) d \xi \\
& =c_{1}^{2} \nu^{-4} \int_{\mathbb{R}^{d}}\left|\widehat{D_{l} f}(\xi)\right|^{2} w_{l}(\xi) d \xi \\
& =c_{1}^{2} \nu^{-4} \int_{\mathbb{R}^{d}}\left|D_{l} f(x)\right|^{2} w_{l}(x) d x \\
& =c_{1}^{2} \nu^{-4}\left\|D_{l} f\right\|_{2, l}^{2} .
\end{aligned}
$$


Therefore

$$
\left\|\left(I-M_{1 / \nu}\right) f\right\|_{2, l} \leq c_{1} \nu^{-2}\left\|D_{l} f\right\|_{2, l} .
$$

Successive applications of (8) to the right-hand side of (7) result in

$$
\begin{aligned}
\left\|f-P_{\nu}(f)\right\|_{2, l} & \leq c_{0}^{-(k+m)} c_{1}^{m} \nu^{-2 m}\left\|\left(I-T_{1 / \nu}\right)^{k} D_{l}^{m} f\right\|_{2, l} \\
& \leq c_{2} \nu^{-2 m} \omega_{k}\left(D_{l}^{m} f, 1 / \nu\right)_{2, l},
\end{aligned}
$$

where $c_{2}=c_{0}^{-(k+m)} c_{1}^{m}$ which implies (5) holds, the theorem is proved.

Proposition 4 The modulus of smoothness $\omega_{k}(f, t)_{2, l}$ has the following properties.

(i) $\omega_{k}(f+g, t)_{2, l} \leq \omega_{k}(f, t)_{2, l}+\omega_{k}(g, t)_{2, l}$.

(ii) $\omega_{k}(f, t)_{2, l} \leq 2^{k}\|f\|_{2, l}$.

(iii) If $f \in W_{2, l}^{k}$, then

$$
\omega_{k}(f, t)_{2, l} \leq c_{3} t^{2 k}\left\|D^{k} f\right\|_{2, l},
$$

where $c_{3}=c_{1}^{k}$ is a constant.

\section{Proof}

Property $(i)$ follow from the definition of $\omega_{k}(f, t)_{2, l}$.

Property (ii) follow from the fact that $\left\|M_{h} f\right\|_{2, l} \leq\|f\|_{2, l}$.

Assume that $h \in(0, t]$. From formulas (1), (3) and Parseval equality, we have

$$
\begin{aligned}
\left\|\Delta_{h}^{k} f\right\|_{2, l}^{2} & =\left\|\widehat{\Delta_{h}^{k} f}\right\|_{2, l}^{2}=\int_{\mathbb{R}^{d}}\left(1-j_{\gamma-\frac{d}{2}-1}(h|\xi|)\right)^{2 k}|\widehat{f}(\xi)|^{2} w_{l}(\xi) d \xi \\
\left\|D_{l}^{k} f\right\|_{2, l}^{2} & =\left\|\widehat{D_{l}^{k} f}\right\|_{2, l}^{2}=\int_{\mathbb{R}^{d}}|\xi|^{4 k}|\widehat{f}(\xi)|^{2} w_{l}(\xi) d \xi
\end{aligned}
$$

Formula (9) implies the equality

$$
\left\|\Delta_{h}^{k} f\right\|_{2, l}^{2}=h^{4 k} \int_{\mathbb{R}^{d}} \frac{\left(1-j_{\gamma-\frac{d}{2}-1}(h|\xi|)\right)^{2 k}}{h^{4 k}|\xi|^{4 k}}|\xi|^{4 k}|\widehat{f}(\xi)|^{2} w_{l}(\xi) d \xi .
$$

From Proposition 2 and Parseval equality we obtain

$$
\begin{aligned}
\left\|\Delta_{h}^{k} f\right\|_{2, l}^{2} & \leq c_{1}^{2 k} h^{4 k} \int_{\mathbb{R}^{d}}|\xi|^{4 k}|\widehat{f}(\xi)|^{2} w_{l}(\xi) d \xi \\
& =c_{1}^{2 k} h^{4 k}\left\|\widehat{D_{l}^{k} f}\right\|_{2, l}=c_{1}^{2 k} h^{4 k}\left\|D_{l}^{k} f\right\|_{2, l}^{2} .
\end{aligned}
$$

Therefore

$$
\left\|\Delta_{h}^{k} f\right\|_{2, l} \leq c_{1}^{k} h^{2 k}\left\|D_{l}^{k} f\right\|_{2, l} .
$$

Calculating the supremum with respect to all $h \in(0, t]$, we obtain

$$
\omega_{k}(f, t)_{2, l} \leq c_{3} t^{2 k}\left\|D_{l}^{k} f\right\|_{2, l},
$$

where $c_{3}=c_{1}^{k}$. 


\section{Proofs of the inverse theorems}

Proposition 5 For $j \geq 1$ we have

$$
2^{2 k(j-1)} E_{2^{j}}(f)_{2, l} \leq \sum_{\eta=2^{j-1}+1}^{2^{j}} \eta^{2 k-1} E_{\eta}(f)_{2, l} .
$$

Proof Note that

$$
\sum_{\eta=2^{j-1}+1}^{2^{j}} \eta^{2 k-1} \geq\left(2^{j-1}\right)^{2 k-1} 2^{j-1}=2^{2 k(j-1)} .
$$

Since $E_{\eta}(f)_{2, l}$ is monotonically decreasing, we conclude that

$$
2^{2 k(j-1)} E_{2^{j}}(f)_{2, l} \leq \sum_{\eta=2^{j-1}+1}^{2^{j}} \eta^{2 k-1} E_{\eta}(f)_{2, l} .
$$

Proposition 6 For $n \in \mathbb{N}$ we have

$$
2^{k} E_{n}(f)_{2, l} \leq \frac{c_{4}}{n^{2 k}} \sum_{j=0}^{n}(j+1)^{2 k-1} E_{j}(f)_{2, l} .
$$

Proof Note that

$$
\sum_{j=0}^{n}(j+1)^{2 k-1} \geq \sum_{j \geq \frac{n}{2}-1}^{n}(j+1)^{2 k-1} \geq\left(\frac{n}{2}\right)^{2 k-1} \frac{n}{2}=2^{-2 k} n^{2 k} .
$$

Since $E_{j}(f)_{2, l}$ is monotonically decreasing, we conclude that

$$
2^{k} E_{n}(f)_{2, l} \leq \frac{c_{4}}{n^{2 k}} \sum_{j=0}^{n}(j+1)^{2 k-1} E_{j}(f)_{2, l} .
$$

Proposition 7. If $\Phi_{\nu} \in I_{\nu}$ such that $\left\|f-\Phi_{\nu}\right\|_{2, l}=E_{\nu}(f)_{2, l}$ For every $\nu \in \mathbb{N}$, then

$$
\left\|D_{l}^{k} \Phi_{2^{j+1}}-D_{l}^{k} \Phi_{2^{j}}\right\|_{2, l} \leq 2^{2 k(j+1)+1} E_{2^{j}}(f)_{2, l} .
$$

In particular

$$
\left\|D_{l}^{k} \Phi_{1}\right\|_{2, l}=\left\|D_{l}^{k} \Phi_{1}-D_{l}^{k} \Phi_{0}\right\|_{2, l} \leq 2^{4 k+1} E_{0}(f)_{2, l} .
$$

Proof By Theorem 1 and the fact that $E_{\nu}(f)_{2, l}$ is monotone decreasing with respect to $\nu$, we obtain

$$
\begin{aligned}
\left\|D_{l}^{k} \Phi_{2^{j+1}}-D_{l}^{k} \Phi_{2^{j}}\right\|_{2, l} & \leq 2^{2 k(j+1)}\left\|\Phi_{2^{j+1}}-\Phi_{2^{j}}\right\|_{2, l} \\
& =2^{2 k(j+1)}\left\|\left(f-\Phi_{2^{j}}\right)-\left(f-\Phi_{2^{j+1}}\right)\right\|_{2, l} \\
& \leq 2^{2 k(j+1)}\left(E_{2^{j}}(f)_{2, l}+E_{2^{j+1}}(f)_{p, \alpha}\right)_{2, l} \\
& \leq 2^{2 k(j+1)+1} E_{2^{j}}(f)_{2, l} .
\end{aligned}
$$


and

$$
\begin{aligned}
\left\|D_{l}^{k} \Phi_{1}-D_{l}^{k} \Phi_{0}\right\|_{2, l} & \leq\left\|\Phi_{1}-\Phi_{0}\right\|_{2, l}=\left\|\left(f-\Phi_{1}\right)-\left(f-\Phi_{0}\right)\right\|_{2, l} \\
& \leq E_{1}(f)_{2, l}+E_{0}(f)_{2, l} \\
& \leq 2 E_{0}(f)_{2, l} \leq 2^{4 k+1} E_{0}(f)_{2, l} .
\end{aligned}
$$

The following theorems are analogues of the classical inverse theorems of approximation theory $[2,5]$.

Theorem 3 For every function $f \in L_{l}^{2}\left(\mathbb{R}^{d}\right)$ and every positive integer $n$ we have

$$
\omega_{k}\left(f, \frac{1}{n}\right)_{2, l} \leq \frac{c}{n^{2 k}} \sum_{j=0}^{n}(j+1)^{2 k-1} E_{j}(f)_{2, l},
$$

where $c=c(k, \alpha)$ is a positive constant.

Proof

Let $2^{m} \leq n<2^{m+1}$ for any integer $m \geq 0$.

For every $\nu \geq 0$, let $\Phi_{\nu}$ be an element of best approximation to $f$ in the space $I_{\nu}$, that is, $\Phi_{\nu} \in I_{\nu}$ and $\left\|f-\Phi_{\nu}\right\|_{2, l}=E_{\nu}(f)_{2, l}$. By formulas $(i)$ and (ii) of Proposition 4, we obtain

$$
\begin{aligned}
\omega_{k}\left(f, \frac{1}{n}\right)_{2, l} & \leq \omega_{k}\left(f-\Phi_{2^{m+1}}, \frac{1}{n}\right)_{2, l}+\omega_{k}\left(\Phi_{2^{m+1}}, \frac{1}{n}\right)_{2, l} \\
& \leq 2^{k}\left\|f-\Phi_{2^{m+1}}\right\|_{2, l}+\omega_{k}\left(\Phi_{2^{m+1}}, \frac{1}{n}\right)_{2, l} .
\end{aligned}
$$

Therefore

$$
\omega_{k}\left(f, \frac{1}{n}\right)_{2, l} \leq 2^{k} E_{2^{m+1}}(f)_{2, l}+\omega_{k}\left(\Phi_{2^{m+1}}, \frac{1}{n}\right)_{2, l} \leq 2^{k} E_{n}(f)_{2, l}+\omega_{k}\left(\Phi_{2^{m+1}}, \frac{1}{n}\right)_{2, l} .
$$

Now with the aid of Proposition 5 and 7 and formula (iii) of Proposition 3, we conclude that

$$
\begin{aligned}
\omega_{k}\left(\Phi_{2^{m+1}}, \frac{1}{n}\right)_{2, l} & \leq \frac{c_{3}}{n^{2 k}}\left\|B^{k} \Phi_{2^{m+1}}\right\|_{2, l} \\
& \leq \frac{c_{3}}{n^{2 k}}\left(\left\|B^{k} \Phi_{1}-B^{k} \Phi_{0}\right\|_{2, l}+\sum_{j=0}^{m}\left\|B^{k} \Phi_{2^{j+1}}-B^{k} \Phi_{2^{j}}\right\|_{2, l}\right) \\
& \leq \frac{c_{3}}{n^{2 k}}\left(2^{4 k+1} E_{0}(f)_{2, l}+\sum_{j=0}^{m} 2^{2 k(j+1)+1} E_{2^{j}}(f)_{2, l}\right) \\
& \leq \frac{c_{3}}{n^{2 k}} 2^{4 k+1}\left(E_{0}(f)_{2, l}+\sum_{j=0}^{m} 2^{2 k(j-1)} E_{2^{j}}(f)_{2, l}\right) \\
& \leq \frac{c_{3}}{n^{2 k}} 2^{4 k+1}\left(E_{0}(f)_{2, l}+E_{1}(f)_{2, l}+\sum_{j=1}^{m} \sum_{\eta=2^{j-1}+1}^{2^{j}} \eta^{2 k-1} E_{\eta}(f)_{2, l}\right) \\
& \leq \frac{c_{3}}{n^{2 k}} 2^{4 k+1}\left(E_{0}(f)_{2, l}+E_{1}(f)_{2, l}+\sum_{j=2}^{2^{m}}(j+1)^{2 k-1} E_{j}(f)_{2, l}\right) .
\end{aligned}
$$


Therefore

$$
\omega_{k}\left(\Phi_{2^{m+1}}, \frac{1}{n}\right)_{2, l} \leq \frac{c_{5}}{n^{2 k}} \sum_{j=0}^{2^{m}}(j+1)^{2 k-1} E_{j}(f)_{2, l} .
$$

Thus from (11) and (12) we derive the estimate

$$
\omega_{k}\left(f, \frac{1}{n}\right)_{2, l} \leq 2^{k} E_{n}(f)_{2, l}+\frac{c_{5}}{n^{2 k}} \sum_{j=0}^{n}(j+1)^{2 k-1} E_{j}(f)_{2, l} .
$$

By Proposition 6 and formula (13), we have

$$
\omega_{k}\left(f, \frac{1}{n}\right)_{2, l} \leq \frac{c}{n^{2 k}} \sum_{j=0}^{n}(j+1)^{2 k-1} E_{j}(f)_{2, l} .
$$

Theorem 4 Suppose that $f \in L_{l}^{2}\left(\mathbb{R}^{d}\right)$ and

$$
\sum_{j=1}^{\infty} j^{2 m-1} E_{j}(f)_{2, l}<\infty .
$$

Then $f \in W_{2, l}^{m}$ and, for every positive integer $n$, we have

$$
\omega_{k}\left(B^{m} f, \frac{1}{n}\right)_{2, l} \leq C\left(\frac{1}{n^{2 k}} \sum_{j=0}^{n}(j+1)^{2(k+m)-1} E_{j}(f)_{2, l}+\sum_{j=n+1}^{\infty} j^{2 m-1} E_{j}(f)_{2, l}\right),
$$

where $C=c(k, m, \alpha)$ is a positive constant.

Proof.

Let $2^{m} \leq n<2^{m+1}$ for any integer $m \geq 0$. For every positive integer $r \leq m$, we consider the series

$$
D_{l}^{r} \Phi_{1}+\sum_{j=0}^{\infty}\left(D_{l}^{r} \Phi_{2^{j+1}}-D_{l}^{r} \Phi_{2^{j}}\right) .
$$

It follows from Propositions 7 and 5 that the series (14) converges in the norm of $L_{l}^{2}\left(\mathbb{R}^{d}\right)$ because

$$
\begin{aligned}
& \sum_{j=0}^{\infty}\left\|D_{l}^{r} \Phi_{2^{j+1}}-D_{l}^{r} \Phi_{2^{j}}\right\|_{2, l} \leq \sum_{j=0}^{\infty} 2^{2 r(j+1)+1} E_{2^{j}}(f)_{2, l} \\
& =2^{2 r+1} E_{1}(f)_{2, l}+c_{2} 2^{4 r+1} \sum_{j=1}^{\infty} 2^{2 r(j-1)} E_{2^{j}}(f)_{2, l} \leq 2^{4 r+1}\left(E_{1}(f)_{2, l}+\sum_{j=1}^{\infty} 2^{2 r(j-1)} E_{2^{j}}(f)_{2, l}\right) \\
& \leq 2^{4 r+1}\left(E_{1}(f)_{2, l}+\sum_{j=1}^{\infty} \sum_{\eta=2^{j-1}+1}^{2^{j}} \eta^{2 r-1} E_{\eta}(f)_{2, l}\right) \\
& \leq 2^{4 r+1} \sum_{j=1}^{\infty} j^{2 r-1} E_{j}(f)_{2, l}<\infty
\end{aligned}
$$


Note that

$$
f=\Phi_{1}+\sum_{j=0}^{\infty}\left(\Phi_{2^{j+1}}-\Phi_{2^{j}}\right)
$$

where the series $(15)$ converges in $L_{l}^{2}\left(\mathbb{R}^{d}\right)$ and, a fortiori, in the space $\mathcal{D}^{\prime}$ of distributions. Since the operator $D_{l}$ is a linear continuous operator on $\mathcal{D}^{\prime}$, the equality

$$
D_{l}^{r} f=D_{l}^{r} \Phi_{1}+\sum_{j=0}^{\infty}\left(D_{l}^{r} \Phi_{2^{j+1}}-D_{l}^{r} \Phi_{2^{j}}\right)
$$

holds in the space $\mathcal{D}^{\prime}$. Since the right-hand side of (16) belongs to $L_{l}^{2}\left(\mathbb{R}^{d}\right)$ for $r \leq m$, we see that $f$ belongs to the Sobolev space $W_{2, l}^{m}$. In particular, $D_{l}^{m} f \in L_{l}^{2}\left(\mathbb{R}^{d}\right)$.

By formula $(i)$ of Proposition 4, we obtain

$$
\omega_{k}\left(D_{l}^{m} f, \frac{1}{n}\right)_{2, l} \leq \omega_{k}\left(D_{l}^{m} f-D_{l}^{m} \Phi_{2^{s+1}}, \frac{1}{n}\right)_{2, l}+\omega_{k}\left(D_{l}^{m} \Phi_{2^{s+1}}, \frac{1}{n}\right)_{2, l} .
$$

Using Propositions 4, 5 and 7 we get

$$
\begin{aligned}
\omega_{k}\left(D_{l}^{m} f-D_{l}^{m} \Phi_{2^{s+1}}, \frac{1}{n}\right)_{2, l} & \leq 2^{k}\left\|D_{l}^{m} f-D_{l}^{m} \Phi_{2^{s+1}}\right\|_{2, l} \\
& \leq 2^{k} \sum_{j=s+1}^{\infty}\left\|D_{l}^{m} \Phi_{2^{j+1}}-D_{l}^{m} \Phi_{2^{j}}\right\|_{2, l} \\
& \leq 2^{k} \sum_{j=s+1}^{\infty} 2^{2 m(j+1)+1} E_{2^{j}}(f)_{2, l} \\
& \leq 2^{k+4 m+1} \sum_{j=s+1}^{\infty} 2^{2 m(j-1)} E_{2^{j}}(f)_{2, l} \\
& \leq 2^{k+4 m+1} \sum_{j=s+1}^{\infty} \sum_{\eta=2^{j-1}+1}^{\infty} \eta^{2 m-1} E_{\eta}(f)_{2, l} \\
& \leq 2^{k+4 m+1} \sum_{j=2^{s}+1}^{\infty} j^{2 m-1} E_{j}(f)_{2, l} .
\end{aligned}
$$

Therefore

$$
\omega_{k}\left(D_{l}^{m} f-D_{l}^{m} \Phi_{2^{s+1}}, \frac{1}{n}\right)_{2, l} \leq c_{6} \sum_{j=2^{s}+1}^{\infty} j^{2 m-1} E_{j}(f)_{2, l}
$$

Now with the aid of Propositions 5 and 7 and by formula (iii) of Proposition 4, we conclude 
that

$$
\begin{aligned}
\omega_{k}\left(D_{l}^{m} \Phi_{2^{s+1}}, \frac{1}{n}\right)_{2, l} & \leq \frac{c_{3}}{n^{2 k}}\left\|D_{l}^{m+k} \Phi_{2^{s+1}}\right\|_{2, l} \\
& \leq \frac{c_{3}}{n^{2 k}}\left(\left\|D_{l}^{m+k} \Phi_{1}-D_{l}^{m+k} \Phi_{0}\right\|_{2, l}+\sum_{j=0}^{s}\left\|D_{l}^{m+k} \Phi_{2^{j+1}}-D_{l}^{m+k} \Phi_{2^{j}}\right\|_{2, l}\right) \\
& \leq \frac{c_{3}}{n^{2 k}}\left(2^{4(k+m)+1} E_{0}(f)_{2, l}+\sum_{j=0}^{s} 2^{2(k+m)(j+1)+1} E_{2^{j}}(f)_{2, l}\right) \\
& \leq \frac{c_{3}}{n^{2 k}} 2^{4(k+m)+1}\left(E_{0}(f)_{2, l}+\sum_{j=0}^{s} 2^{2(k+m)(j-1)} E_{2^{j}}(f)_{2, l}\right) \\
& \leq \frac{c_{3}}{n^{2 k}} 2^{4(k+m)+1}\left(E_{0}(f)_{2, l}+E_{1}(f)_{2, l}+\sum_{j=1}^{s} \sum_{\eta=2^{j-1}+1}^{2^{j}} \eta^{2(k+m)-1} E_{\eta}(f)_{2, l}\right) \\
& \leq \frac{c_{3}}{n^{2 k}} 2^{4(k+m)+1}\left(E_{0}(f)_{2, l}+E_{1}(f)_{2, l}+\sum_{j=2}^{2^{s}}(j+1)^{2(k+m)-1} E_{j}(f)_{2, l}\right)
\end{aligned}
$$

Therefore

$$
\omega_{k}\left(D_{l}^{m} \Phi_{2^{s+1}}, \frac{1}{n}\right)_{2, l} \leq \frac{c_{7}}{n^{2 k}} \sum_{j=0}^{2^{s}}(j+1)^{2(k+m)-1} E_{j}(f)_{2, l} .
$$

Thus from (17) and (18) we derive the estimate

$$
\omega_{k}\left(D_{l}^{m} f, \frac{1}{n}\right)_{2, l} \leq C\left(\sum_{j=n+1}^{\infty} j^{2 m-1} E_{j}(f)_{2, l}+\frac{1}{n^{2 k}} \sum_{j=0}^{n}(j+1)^{2(k+m)-1} E_{j}(f)_{2, l}\right) .
$$

\section{Acknowledgemnt}

The authors would like to thank the referee for his valuable comments and suggestions.

\section{References}

[1] Bernstein, S. Sur l'ordre de la meilleure approximation des fonctions continues par des polynomes de degrè donnè. Mem.Acad.Roy.Belgigue, 2 me sèrie. 1912. 4: 1-104.

[2] Stechkin, S. B. On the order of best approximation of continuous functions (Russian). Izv.Akad.Nauk.SSR, Ser.Math. 1954. 15: 219-143.

[3] Timan, M. F. Best approximation and modulus of smoothness of functions prescribed on the entier real axis, (Russian). Izv.Vyssh. Uchebn. Zaved Matematika. 1961. 25: $108-120$ 
[4] Platonov, S. S. Approximation of function in $L_{2}$-metric on noncompact rank 1 symmetric spaces. Algebra in Analiz. 1999. 11(1): 244-270

[5] Timan, A. F. Theory of approximation of functions of a real variable, English transl. New York: Dover Publications, Inc. 1994.

[6] Jeu, M. F. The Dunkl transform. Invent. Math. 1993. 113: 147-162.

[7] Dunkl, C. F. Hankel transform associated to finite reflection groups, Hypergeometric functions on domains of positivity, Jak polynomials and applications. Contemp. Math. 1992. 138: 123-138.

[8] Rösler, M. Positivit- of the Dunkl intertwining operator, Dunke Math. J. 98(3) (1999) 445-463.

[9] Dunkl, C. F. Integral kernels with reflection group invariance. Canadian J. Math. 1991. 43: $1213-1227$.

[10] Kolmogorov, A. N and Fomin, S. V. Elements of theory of functions and functional analysis. Moscow: Nauka. 1976 [in Russian].

[11] Rösler, M. Generalized hermite polynomials and the heat equation for Dunkl operators. Comm. Math. Phy. 1998. 192: 519-542 arXiv:9703006.

[12] Trimèche, K. Paley-Wiener theorems for Dunkl transform and Dunkl translation operators. Integral Transforms Spec. Funct. .2002. 13: 17-38.

[13] Bateman, H and Erdélyi. Higher transcendental functions. (New York: McGraw-Hill. 1953) (vol. II. Moscow: Nauka, 1974).

[14] Abilov, V. A and Abilova, F. V. Approximation of functions by Fourier-Bessel Sums. Izv. Vyssh. Uchebn. Zaved. Math. 2001. 8: 3-9.

[15] Belkina, E. S. and Platonov, S. S. Equivalence of k-functionnals and modulus of smoothness constructed by generalized dunkl translations. Izv. Vyssh.Uchebn. Zaved. Mat. 2008. 8: 3-15.

[16] Maslouhi, M. An analog of Titchmarsh's thorem for Dunkl transform. Integral Transform Spec Funct. 2010. 21(10): 771-778. 